\title{
The effect of inhomogenities on single molecule imaging by hard XFEL pulses
}

\author{
Zoltán Jurek and Gyula Faigel \\ Research Institute for Solid State Physics and Optics \\ H 1525 Budapest, $P O B$ 49, Hungary
}

(Dated: November 1, 2018)

\begin{abstract}
We study the local distortion of the atomic structure in small biological samples illuminated by $\mathrm{x}$ ray free electron laser (XFEL) pulses. We concentrate on the effect of inhomogenities: heavy atoms in a light matrix and non-homogeneous spatial distribution of atoms. In biological systems we find both. Using molecular-dynamics type modeling it is shown that the local distortions about heavy atoms are larger than the average distortion in the light matrix. Further it is also shown that the large spatial density fluctuations also significantly alter the time evolution of atomic displacements as compared to samples with uniform density. This fact has serious consequences on single particle imaging. This is discussed and the possibility of a correction is envisaged.
\end{abstract}

PACS numbers: 87.15.ap, 87.15.B-, 87.53.-j, 61.80.Lj, 52.65.Yy 


\section{INTRODUCTION}

With the introduction of XFEL sources new possibilities open for structural studies. This is clearly illustrated by the several works done on the already existing soft XFEL-s [1, 2, 3]. In the near future similar sources in the hard x-ray regime will also be operational. According to the work of Neutze et al [4] the very short and intense x-ray pulses of XFEL-s might allow atomic resolution imaging of single non-periodic objects. Their idea is that one collects the elastically scattered photons before the deterioration of the sample is appreciable. Many models have been worked out for the description of the behavior of the sample in the XFEL pulse [5, 6, 7]. Their conclusion is that at $\sim 10 \mathrm{keV}$ the $\sim 10^{13}$ photons have to be concentrated in an approximately $10 \mathrm{fs}$ time window for successful atomic resolution imaging. Sources presently under construction do not meet simultaneously all the requirements. Therefore various ideas are introduced to relax the above conditions. One of them is the application of a thin sacrificial tamper layer about the sample [6, 8, 9$]$. In this case the sample deteriorates with a slower rate, which may allow longer times for imaging. However, this layer adds to the background, which might hinder structure solution [9, 10]. Other suggestions concentrate to the evaluation process [9, 11]: in one of them [9] the possibility to correct the charge loss during imaging is pointed out, and in the other a new method for the construction of the 3D reciprocal space dataset is introduced [11]. To understand the usefulness and limits of the various approaches one should start from as realistic description of the damage process as possible. So far most of the modeling was done for spatially homogeneous samples. The reason is that this assumption makes the modeling simpler, more tractable. This allowed the application of continuum models, with reduced dimensionality (1D) [6, 8]. Even more sophisticated models like Molecular Dynamics type models (MD) were applied for homogeneous systems, mostly because of comparability with the results of continuum models [5, 9]. However, MD modeling allows the description of more realistic non-homogeneous systems like biological molecules. Further, it has an other advantage; it directly gives atomic coordinates, which are necessary for the calculation of the diffraction pattern. In this paper we concentrate on the effect of inhomogenities on sample dynamics. Inhomogenity is inherent to biological systems. In these systems we find short and long near neighbor distances leading to spatially non-homogeneous arrangement of atoms. Further, in many cases we also find heavy elements in the light matrix. A typical example is iron, which 
is contained by various building blocks of living organisms. However, many other heavy atoms $(\mathrm{Co}, \mathrm{Ni}, \mathrm{I}$ etc.) can also be found in biological systems. In the present work we show that the local distortion about heavy atoms is much larger than the average distortion in a biological sample. It is also shown that the large spatial density fluctuations significantly alter the time evolution of the atomic displacements. This fact was not recognized until now. We discuss our results from the point of view of structural imaging. We use simple $\mathrm{C}$ and $\mathrm{C}-\mathrm{Fe}$ model systems to separately illustrate the above points, and we also show the deterioration of a real biological system, the myoglobin, in which the two effects appear at the same time. Based on our model-calculations it might be possible to correct for the above distorting effects.

\section{MODEL PARAMETERS AND MODEL-SAMPLES}

In the proposed experiment, individual identical molecules are going to be exposed to XFEL pulses one-by-one in random, unknown orientation. Estimates for the number of elastically scattered photons show, that single diffraction patterns will be very noisy. Therefore the compilation of a 3D diffraction pattern, which is necessary to solve the structure, is not possible directly from the individual 2D images. Many pictures have to be collected and added to improve statistics. This means that the minimum requirement of photon counting statistics is determined by the ability to classify the diffraction patterns according to their orientations. The requirements set by the classification might be relaxed according to a new approach, which uses the collected data set as a whole [11]. However, the applicability of this approach is not proved for a realistic large data set, therefore we use the more conservative estimate given by the classification [10, 12]. Based on [10, 12] we are considering XFEL pulses with energy of $12 \mathrm{keV}$ and with fluence $10^{13} \mathrm{ph} / \mathrm{pulse}$. This beam is focused to a $100 \mathrm{~nm}$ diameter spot. As pulse length we use $10 \mathrm{fs}$ with flat top shape, which is shorter than the pulse length of XFEL-s under construction but it is realistically reachable. The reason of using this short pulse is that even under this short time, atoms might move appreciable distances. In our MD model the motion of all particles are followed by solving the non-relativistic equations of motion. Coulomb forces are explicitly included, and the various quantum-processes are taken into account through their cross sections as stochastic

processes. The detailed description of this model is given in [5]. As a reference model-system 
(a)

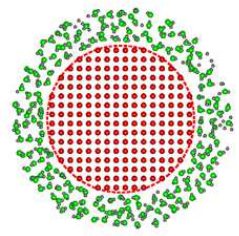

(c)

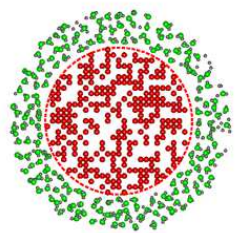

(b)

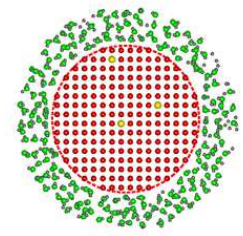

(d)

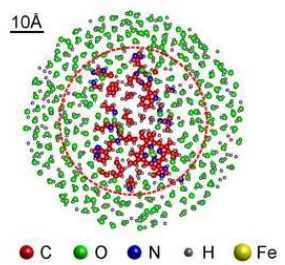

FIG. 1: Starting structures used in the model-calculations. For clarity we show four atomic layers thick cross sections of the samples: homogeneous $40 \AA$ diameter pure Carbon sample (a); the same sample as in (a) but 3 carbon atoms replaced by Fe (b); spatially non-homogeneous sample (c), and myoglobin (d). All samples are surrounded by a water layer resulting in spherical droplets with $60 \AA$ total diameters. The sample parts are encircled by $40 \AA$ diameter spheres. The color convention given in the figure is used through the paper.

we use a $40 \AA$ diameter carbon cluster with homogeneously distributed C-s, surrounded by a $10 \AA$ thick water tamper layer [sample (A)] (Fig. 1,a.). Atoms are placed on a $2.5 \AA$ grid, which corresponds to the typical density of biological systems, $1.35 \mathrm{~g} / \mathrm{cm}^{3}$. In order to illustrate the effect of heavy atoms, the same $40 \AA$ diameter carbon cluster is used with three carbons exchanged to Fe [sample (B)] (Fig. 1,b.). The effect of spatial inhomogenities is studied on a pure carbon system with similar sizes and average density as in the previous cases, but in this sample the density is not spatially homogeneous [sample (C)]. The sample is built in the following way: first $\mathrm{C}$ atoms are placed on a dense regular grid $a=1.5 \AA$. This leads to $6.25 \mathrm{~g} / \mathrm{cm}^{3}$ density. To reach the $1.35 \mathrm{~g} / \mathrm{cm}^{3}$, randomly chosen atoms are removed (Fig. 1.c). At last the behaviour of a real biological molecule, the myoglobin is modeled [sample (D)] (Fig. 1,d ). 

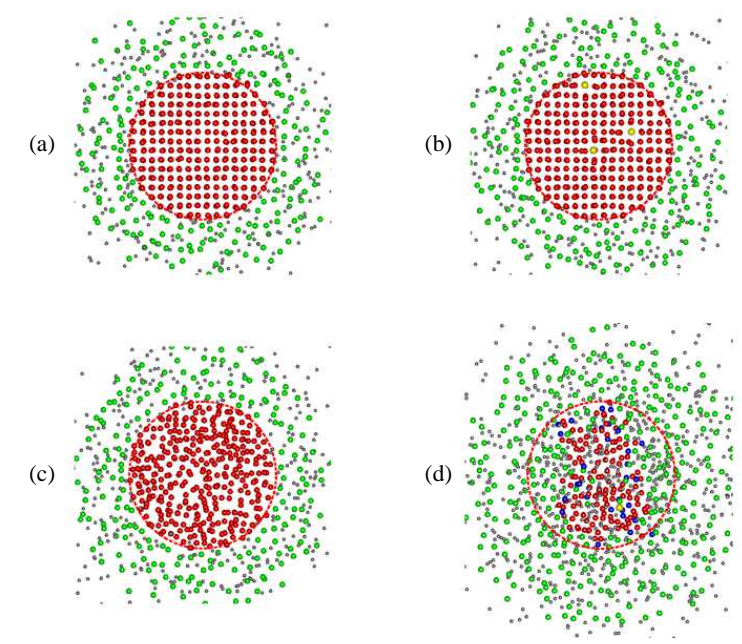

FIG. 2: The structure of $\mathrm{C}(\mathrm{a}), \mathrm{Fe}-\mathrm{C}(\mathrm{b})$, non-homogeneous $\mathrm{C}(\mathrm{c})$ and myoglobin (d) at the end of the pulse.

\section{RESULTS}

First we discuss the effect of heavy atoms. On Fig. 2, a and b the atomic positions in the $\mathrm{C}$ and $\mathrm{C}-\mathrm{Fe}$ systems are shown at the end of the pulse. For easy comparison not the whole samples, only their cross sections are depicted. Further, we do not show the electrons for clarity. In the case of the pure carbon system [sample (A)] the changes in the structure are hardly recognizable. However, in the water layer there are significant distortions. The reason of the different behavior of the sample and of the water parts were discussed in previous works [8, 9]. Here we briefly describe the processes leading to this particular behaviour. This discussion also helps to understand the reasoning behind the effects of heavy atoms and spatial inhomogenities. When the probe beam hits the sample, high energy photoelectrons are produced. These leave the sample resulting in highly excited ions and a positively charged particle. Auger relaxation and electron-ion collisions lead to a large number of free electrons. These non-bonded electrons rapidly rearrange in such a way, that a neutral core - composed of ions and electrons - develops. In the core there is an effective Debye shielding, which slows down the deterioration of the sample. Around the core we find a positively charged layer, in which the Coulomb forces are not shielded resulting in the fast explosion of this layer. In order to avoid large structural distortion of the sample the thickness of the water layer has to be chosen to be equal to the thickness of the positively 

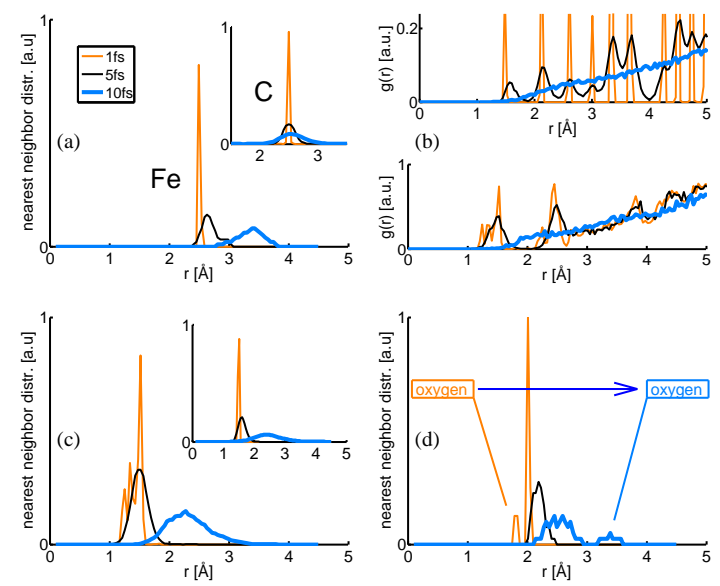

FIG. 3: Time evolution of the first neighbor distributions and pair correlation functions. (a) first neighbor distributions around the Fe and $\mathrm{C}$ atoms (inset) in sample (B). (b) pair correlation functions of sample (C) (upper panel) and (D) (lower panel). (c) First neighbor distributions around the $\mathrm{C}$ atoms in sample (D) and (C) (inset). (d) First neighbor distribution around the Fe in sample (D).

charged shell. In our case $10 \AA$ was chosen as the minimum necessary thickness. Until this point our results agree with those of the continuum models [6, 8]. Now we turn to the Fe-C sample. Inspecting Fig. 2.b. it is clear, that a significantly larger distortion develops around the $\mathrm{Fe}$ atoms than around the matrix $\mathrm{C}$ atoms. To make this difference more quantitative we plotted the first nieghbour distributions about $\mathrm{Fe}$ and $\mathrm{C}$ atoms on Fig. 3. a. In the case of Fe centers the distortion is large; the center of the distribution shifts about $1 \AA$ as compared to the original atomic positions. Further, the width of the distribution increases to about $1 \AA$. An increase of the width is expected because the random nature of various ionization processes lead to different momentum transfer to the ions resulting in different displacements. For $\mathrm{C}$ atoms, the center of the distribution shifts only around $0.1 \AA$, small compared to the Fe case. The increase of the width of the distribution is about the same as for Fe. We can understand the different behaviour of Fe environment by analyzing the physical processes. The differences come from two sources: (i) the ionization dynamics of the Fe is much faster than that of $\mathrm{C}$, therefore a high ionization state of the Fe atoms develops in a short time; (ii) the Debye shielding does not work effectively for local inhomogenities. The reason is that the electrons shield the average charge of the system, which is determined by the $\mathrm{C}$ ions. The much higher positive charge of the Fe ions is not compensated by the 
collective motion of electrons. These two factors lead to a large Coulomb repulsion about the Fe atoms, which pushes out the neighboring C-s. Next we describe the effect of nonhomogeneous spatial atomic arrangement. On Fig. 2.c. sample (C) is shown at the end of the pulse. We can identify large structural changes. One can follow this quantitatively by analyzing the time dependence of the pair correlation function (Fig. 3.,b., upper panel) and the nearest neighbour distribution (Fig. 3.c. inset). We can divide the nearest neighbours into three classes: $1.5 \AA, 2.1 \AA, 2.55 \AA$. Following these separately, we see a large increase of the peak position of the first class, a much smaller change in the second class and almost nothing in the third class. At the end of the pulse an almost homogeneous system develops. The explanation of this behaviour is the following: the shielding by the free electrons is determined by the average density, which corresponds to $2.5 \AA$ first neighbour distance. In this spatial range the Debye shielding is effective. However, for shorter distances the positive charge of the $\mathrm{C}$ ions are not compensated, which leads to large Coulomb forces between close ions. The second factor, which determines the motion of ions is the available space. In the inhomogeneous system the ions tend to move toward those places, where the local density is small (see Fig. 1.c. and Fig. 2.c). Starting from the above calculations we can understand the behaviour of the myoglobin in the XFEL pulse. In Fig. 2, d. the molecule is shown at the end of the pulse. Its deterioration is severe, it resembles to that of sample (C). Plotting the time evolution of the pair correlation functions (Fig. 3.,b.,lower panel) we see a similar trend than for the model system, with the exception, that one cannot separate three distinct first neighbour distances. This is natural, since in a real molecule the bonding distances change almost continuously according to the various types of bonds. We can obtain even more details by separately studying the $\mathrm{C}$ and Fe environments (Fig. 3.c and d.). While the time evolution of the $\mathrm{C}$ environment is very close to that of the reference system (Fig. 3.c.) the Fe environment shows a slightly different behaviour (Fig. 3.a. and d.). The reason is that in the case of myoglobin the Fe first neighbour distances are smaller than it was in the model system. Therefore the heavy atom effect is combined with the effect of non-homogeneous spatial arrangement. As a result, some elements in the Fe environment move even more than in the model system, and some move less, depending on the interplay between the geometric arrangement and the extra charge on the Fe ion. To illustrate this, we plotted the environment of the Fe ion as a function of time (Fig. 4). The figure clearly shows that the oxygen atoms move the most, while the nitrogen atoms the less. In the light 

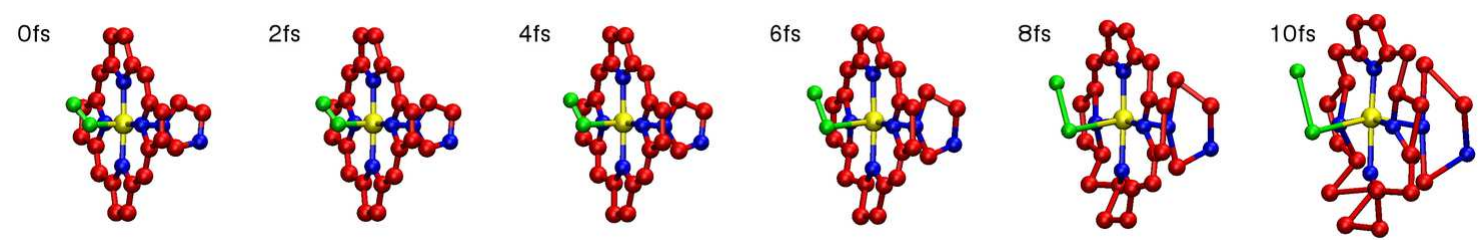

FIG. 4: The $4.5 \AA$ radius environment of the Fe atom in the myoglobin at $0,2,4,6,8$ and 10 fs.

of our calculations this is not surprising since the $\mathrm{Fe}-\mathrm{O}$ distance is the shortest leading to a large Coulomb repulsion. Further, there is a large empty space for the oxygen atoms to move to. In the case of the nitrogen atoms the starting $\mathrm{Fe}-\mathrm{N}$ distance is larger and there is no room around the nitrogen atoms, so their neighbors slow down their motions. At last we point out one more interesting feature. In all cases the volume and the shape of the samples do not change. To emphasize this we put a constant diameter sphere around the samples (Fig. 1, and Fig. 2,). Why is this fact important? This clearly shows that a 1D homogeneous shell model would predict a negligible distortion for all samples, since the Debye shielding, which governs the damage in these models depends on the average density but not on the detailed structure of the samples. However, as our calculations show this is very far from reality. Of course our conclusions can be generalized to other nonbiological systems containing inhomogenities. This fact has serious consequences on single particle imaging. Although the overall shape and size of the samples may remain the same during an XFEL pulse, their internal atomic structure can significantly change. Therefore the reconstruction of the original structure at the atomic level cannot be directly obtained, even slowing down the sample destruction with the application of a tamper layer. This layer slows down the explosion of the sample as a whole, but the internal atomic structure may drastically change.

\section{SUMMARY AND CONCLUSION}

The behaviour of small samples in highly focused XFEL pulses was studied. We have examined the effect of inhomogenities on the time evolution of the distortions of near neighbor environments. It was found that around heavy atoms the local distortions significantly increase as compared to the distortions about the light matrix atoms. An even larger distor- 
tion develops around density fluctuations, where the light atoms are much closer than the average distance corresponding to the density of the system. Both of these effects are present in biological systems, which are one of the target areas of research by XFEL sources. Until now most of the damage modeling was done for homogeneous systems, and conclusions about the feasibility of structural imaging experiments were drawn from these calculations. Our study shows that atomic resolution imaging is hindered by inhomogenities, and no reliable information can be obtained from models using simplified homogeneous density. Unfortunately this problem cannot be overcome by the application of tamper layers, because local distortions show up even in the presence of Debye shielding. However, knowing this type of behavior, we can envisage the introduction of corrections in the evaluation process. It is clear that we cannot avoid the distortions, but we can use the fact that we know about it. We know that diffraction data will result too large first neighbor distances about heavy atom sites, and also about such sites where light atoms are very close to each other. Using MD type modeling we can correct the structure obtained directly from the diffraction data. This could be done in a multi-step process: (i) roughly correct the structure obtained from the diffraction data and use this as input parameters to the MD modeling; (ii) calculate the time evolution of the corrected structure and use this result to calculate the diffraction pattern; (iii) compare this to the measured one and correct the starting structure accordingly; (iv) start again MD modeling with this new structure and continue until the calculated and measured patterns are close enough. Using this type of approach, atomic resolution imaging might be possible even in the presence of large local distortions.

The work reported here was supported by OTKA 67866 and NKFP1/0007/2005 grants.

[1] H. N. Chapman et al, Nature 448, 676 (2007).

[2] J. Hajdu and F. Maia, Nature Physics 4, 351 (2008).

[3] M. J. Bogan et al, Nano Letters 8, 310 (2008).

[4] R. Neutze et al., Nature 406, 752 (2000).

[5] Z. Jurek, G. Faigel, and M. Tegze, Eur. Phys. J. D. 29, 217 (2004).

[6] S. Hau-Riege, R. London, and A. Szoke, Phys. Rev. E 69, 051906 (2004).

[7] M. Bergh, N. Timneanu, and D. van der Spoel, Phys. Rev. E 70, 051904 (2004). 
[8] S. P. Hau-Riege et al., Phys. Rev. Lett. 98, 198302 (2007).

[9] Z. Jurek and G. Faigel, Eur. Phys. J. D 50, 35 (2008).

[10] G. Bortel and G. Faigel, J. of Struct. Biology 158, 10 (2007).

[11] R. Fung et al., Nature Physics 5, 64 (2008).

[12] G. Bortel, G. Faigel, and M. Tegze, J. of Struct. Biology (in press). 